\title{
RIESGOS EN EL NOVIAZGO
}

\author{
Balabarca Cárdenas, Yván \\ Facultad de Teología \\ Universidad Peruana Unión \\ yvanbalabarca@teologia.edu.pe
}

Fecha de recepción: Agosto 2012

Fecha de aceptación y versión final: Septiembre 2012

\section{Resumen}

El desarrollo de programas en la Web 2.0 es uno de los métodos que en la difusión de conductas saludables y la prevención de conductas sexuales de riesgo se está utilizando. En este marco, se desarrolló una encuesta para averiguar en cien internautas cuáles eran los mayores riesgos en el noviazgo. Se utilizaron las plataformas FACEBOOK, USTREAM y ENCUESTA FÁCIL para captar los internautas y sus opiniones. Luego se empleó el software estadístico SPSS v. 20. Los resultados indicaron que las mujeres consideraron que las relaciones sexuales coitales son el principal riesgo en el noviazgo, mientras que los varones consideraron a las caricias intimas como la principal. Esto provee orientación para direccionar el énfasis, en los programas de salud sexual para jóvenes.

Palabras claves: Riesgos, noviazgo, adolescencia, salud social, salud sexual 


\section{Marco Teórico}

Se han desarrollado diversos programas con el fin de prevenir conductas de riesgo en la etapa del noviazgo. En el Perú, los estudios acerca de los riesgos en el noviazgo no son abundantes.

Por otro lado, en otras latitudes se aprecian investigaciones en torno al tema del noviazgo. Peltzer (2006) en un estudio desarrollado con 800 adolescentes (16 o 17 años), hombres y mujeres, para entender el cambio social de los adolescentes en el contexto del VIH/SIDA, en Sudáfrica, encontró que los adolescentes de las zonas urbanas estaban significativamente más relacionados a prácticas como el petting, masturbación, sexo anal y oral, que los adolescentes del área rural. Este hecho destaca una realidad de los adolescentes de áreas urbanas, pues ellos presentan una mayor actividad de sexo coital que los adolescentes de áreas rurales.

Souza y Chamorro (2006), destaca que en nuestros tiempos los medios de comunicación masiva, movidos por intereses mercantiles, difunden una inadecuada influencias cultural que resulta nociva para los consumidores. A esto se suma una grave psicopa- tología y la manipulación de los vectores de la socialización. Ante tal fenómeno los miembros de la sociedad promueven educación para la salud y aspectos psicosociales. Por ello, las sociedades vulnerables exhiben conductas antisociales, autoagresividad y heteroagresividad, y demás "sensaciones fuertes" como deportes extremos, patrones de consumo de psicotrópicos, conducción de vehículos de alta velocidad, promiscuidad y violencia sexual, portación de armas, robos, vandalismo, con miras a aplacar estados emocionales derivados del vacío existencial (frustración e insatisfacción vital, desesperación, enajenación, caos y tendencias suicidas). Se debe tener una participación más enérgica y decidida de todos los que conformamos la sociedad.

En Estados Unidos, el 47.5\% de adolescentes del último año de la preparatoria (secundaria en el Perú), según Eaton, Kann, Kinchen, Shanklin, Flint, Hawkins, \& ...Wechsler, H. han tenido alguna experiencia sexual con al menos una persona. En el Perú, el informe del Instituto Nacional de Estadísticas e Informática (INEI) indicó que las mujeres del área rural inician su vida sexual más temprano que las del área ur- 
bana, y esto es más notorio entre las mujeres de la selva rural, donde el $15.5 \%$ tuvo su primera relación sexual entre los 10 y 14 años, el 55.8\%, entre los 15 y 17 años, el $18.4 \%$, entre los 18 y 19 años y apenas el $10.3 \%$, después de los 20 años de edad.

Se necesita investigar los móviles de estos contactos sexuales tempranos de la mujer rural, ya que no se revelan datos de si fueron consentidos o forzados, por ejemplo; además se precisan investigaciones de tipo cualitativo para ver el trasfondo antropológico y aun cultural de las regiones.

En las áreas urbanas de la Costa y Sierra, así como en Lima Metropolitana las mujeres inician su sexualidad a edades más tardías, es decir, después de los 20 años, siendo en la costa urbana el $40.1 \%$, en la sierra urbana el $38.8 \%$ y en Lima Metropolitana el $40.3 \%$.

Varios modelos explican que la percepción de las consecuencias potenciales de los riesgos sexuales y los beneficios de una conducta de cuidado sexual, juegan un papel importante en la decisión que los adolescentes hacen. De allí la importancia de implementar programas de difusión de conductas sociales saludables en la amistad y el noviazgo durante la adolescencia y la juventud, según Mallet y Herbé (2011).

Por otro lado, un estudio desarrollado en la Universidad de Salamanca, España, sobre el sexismo y las agresiones físicas, sexuales y verbales-emocionales, en relaciones de noviazgo de estudiantes universitarios, se encontraron diferencias significativas en los niveles de sexismo entre hombres y mujeres. Los hombres manifestaron haber perpetrado más agresiones sexuales mientras que las mujeres más agresiones verbales - emocionales. No hubo diferencia significativa en la perpetración de agresiones físicas. En cuanto a agresiones físicas sufridas, los hombres reportaron haber sido más agredidos. No se encontró correlación significativa entre actitudes sexistas y comportamientos agresivos cometidos.

En los programas de intervención psicosocial de salud es menester incluir un componente actitudinal de habilidades psicológicas de resolución de conflictos y autoestima, a fin de que las parejas jóvenes puedan conocer y reconocer las señales de violencia en el desarrollo de su relación y tomen las decisiones saludables adecuadas. 
También en España se desarrolló un estudio para medir los indicadores de violencia en el noviazgo, utilizaron el Cuestionario de Violencia de Novios (CUVINO), que evalúa tanto la frecuencia de conductas violentas como los niveles de molestia asociados a ellas. Lo interesante de este estudio es que los niveles de molestia ante estas conductas abusivas fueron estadísticamente no significativas por parte de la parte agredida. Es necesario orientar sobre la agresión no solo de tipo verbal, sino también física y sexual.

Un dato interesante del estudio de Muñoz-Rivas, Graña, 72 O'Leary y Gonzáles que trató sobre la prevalencia y predoctores de agresión sexual en las relaciones de noviazgo en adolescentes y jóvenes, con una muestras de 4052 adolescentes y jóvenes adultos, fue que tanto la victimización sexual como la agresión son de naturaleza fundamentalmente psicológica; y la victimización sexual se predice en función de la agresión sexual de los individuos estudiados tanto en el caso de los hombres como en el de las mujeres. Un agresor necesita una víctima. Líderes juveniles entrenados en componentes de salud mental se hacen necesarios en el medio educativo.
En un estudio desarrollado en la Universidad de Extremadura, se encontró que los jóvenes universitarios de entre 17 y 23 años describen patrones de interacción coactiva en sus relaciones de noviazgo. Además, quienes ponen en práctica patrones de violencia psicológica, en mayor medida son los jóvenes entre 17 y 18 años. Es necesario adelantarnos orientando en la soltería para que llegue a hacer un buen matrimonio.

\section{Método}

Se hizo uso de las tecnologías de la información y de las comunicaciones, como son: FACE$\mathrm{BOOK}$, además de programas de encuestas y estadísticas como son ENCUESTA FÁCIL y SPSS (v. 20) para MAC, se estructuró una encuesta donde se exploró la edad, género, estado civil, si viven con sus padres, lugar de residencia, edad de su primera relación sentimental y cuál considera que es el riesgo en el noviazgo más grave.

Se propusieron las siguientes opciones: Las caricias íntimas, el aislamiento, el no escuchar consejos, la infidelidad, el sexo prematrimonial, la pornografía, la violencia física o verbal, el uso de sustancias adictivas, noviazgos largos y otros. 
La muestra fue de 100 encuestados, todos voluntarios y que fueron contactados vía el FACE$\mathrm{BOOK}$, tanto del investigador como de voluntarios que difundieron el instrumento a través de sus respectivas cuentas.

\section{Resultados}

Los datos se analizaron a través del software SPSS para MAC, versión 20, a través de tablas de contingencias. Los resultados fueron los siguientes.

\section{Tabla 1:}

\section{Intervalo entre edad y riesgo en el noviazgo}

\begin{tabular}{|c|c|c|c|c|c|c|c|c|c|c|c|}
\hline & \multicolumn{9}{|c|}{ Riesgo en el noviazgo } & \multirow[t]{2}{*}{ Total } \\
\hline & & 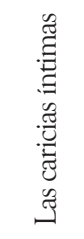 & 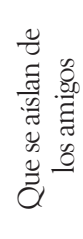 & 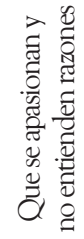 & 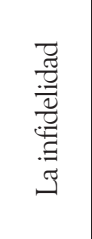 & 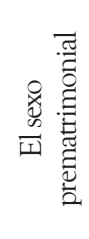 & 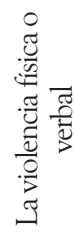 & 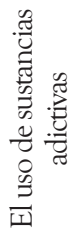 & 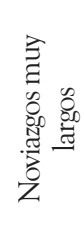 & $\stackrel{8}{0}$ & \\
\hline \multirow{11}{*}{ 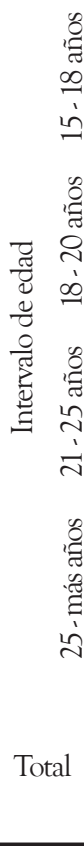 } & \multirow{11}{*}{ 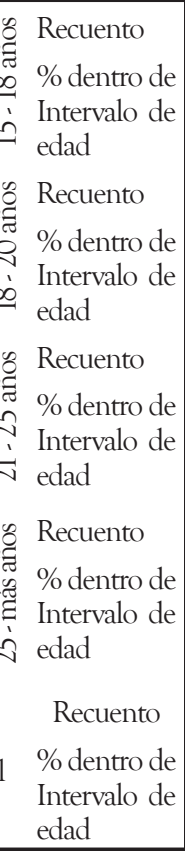 } & 1 & 0 & 1 & 0 & 0 & 0 & 0 & 0 & 0 & 2 \\
\hline & & $50,0 \%$ & $0,0 \%$ & $50,0 \%$ & $0,0 \%$ & $0,0 \%$ & $0,0 \%$ & $0,0 \%$ & $0,0 \%$ & $0,0 \%$ & $100,0 \%$ \\
\hline & & 2 & 1 & 3 & 4 & 5 & 1 & 1 & 0 & 1 & 18 \\
\hline & & $111 \%$ & $56 \%$ & $167 \%$ & \%כ2 2 & $278 \%$ & $56 \%$ & $56 \%$ & ○० & $56 \%$ & $100 \Omega \%$ \\
\hline & & & 5,0\% & $10,1 \%$ & $\angle L, L \%$ & $21,0 \%$ & $5,0 \%$ & 5,0\% &, $0 \%$ & J,0\% & $100,0 \%$ \\
\hline & & 11 & 6 & 4 & 5 & 7 & 1 & 0 & 4 & 1 & 39 \\
\hline & & $28,2 \%$ & $15,4 \%$ & $10,3 \%$ & $12,8 \%$ & $17,9 \%$ & $2,6 \%$ & $0,0 \%$ & $10,3 \%$ & $2,6 \%$ & $100,0 \%$ \\
\hline & & 7 & 1 & 6 & 1 & 16 & 2 & 0 & 6 & 1 & 40 \\
\hline & & $17,5 \%$ & $2,5 \%$ & $15,0 \%$ & $2,5 \%$ & $40,0 \%$ & $5,0 \%$ & $0,0 \%$ & $15,0 \%$ & $2,5 \%$ & $100,0 \%$ \\
\hline & & 21 & 8 & 14 & 10 & 28 & 4 & 1 & 10 & 3 & 99 \\
\hline & & $21,2 \%$ & $8,1 \%$ & $14,1 \%$ & $10,1 \%$ & $28,3 \%$ & $4,0 \%$ & $1,0 \%$ & $10,1 \%$ & $3,0 \%$ & $100,0 \%$ \\
\hline
\end{tabular}


En la Tabla 1 los jóvenes de entre 18 y 20 años que participaron de la encuesta refirieron en un $27,8 \%$ que la práctica del sexo prematrimonial es el primer riesgo en el noviazgo. Se deja entrever su conocimiento sobre las consecuencias propias de esta práctica de riesgo antes del matrimonio, a saber, embarazos no deseados, infecciones de transmisión sexual (SIDA), y el aborto con sus complicaciones.
Por otro lado, los jóvenes de entre 21 y 25 años, en un $28,2 \%$, consideraron que el mayor riesgo son las caricias íntimas, quizá debido a que consideran que son el preámbulo de las relaciones cóitales, o del sexo anal u oral. De ser así, este grupo etáreo opta por una prevención total, quizá por los casos de los que han tenido conocimiento, construyen una mayor área de prevención que los más jóvenes.

\section{Tabla 2:}

\section{Intervalo entre sexo y riesgo en el noviazgo}

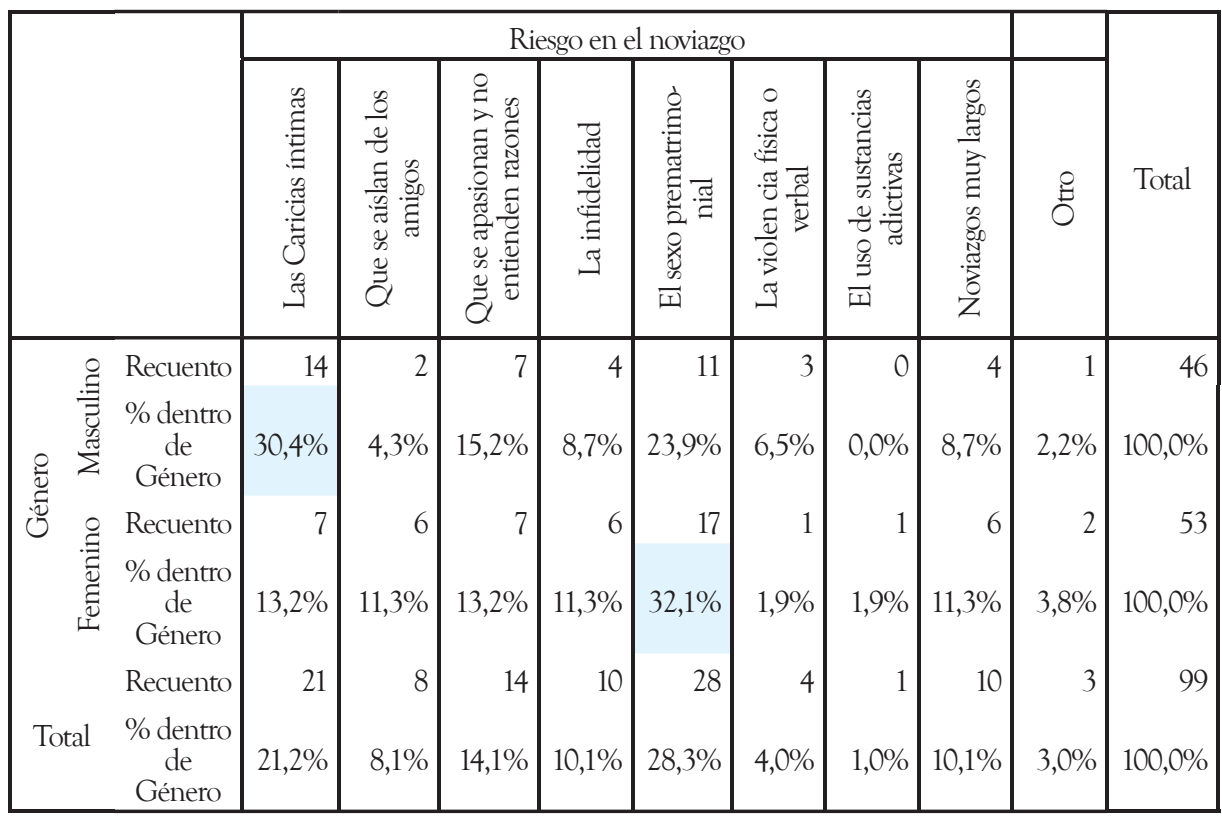


La Tabla 2 nos permite varias lecturas, la primera es que los hombres consideran como de mayor riesgo a las caricias íntimas $(30,4 \%)$, y en segundo lugar el sexo prematrimonial $(23,9 \%)$, mientras que las mujeres, de forma contraria, les preocupa más el sexo prematrimonial $(32,1 \%)$ que las caricias íntimas (13,2\%); algo que les parece riesgoso a ambos grupos es que las caricias íntimas anulen el juicio crítico.

Si consideramos que las caricias íntimas preceden al sexo coital, entonces quienes tienen mayor noción de prevención son los varones. Quizá se deba a que las mujeres valoran la virginidad. Sin embargo, esto da pie a desarrollar una investigación de tipo cualitativo a fin de conocer percepciones y trasfondos culturales arraigados.

La Tabla 3 deja ver que un mayor número de damas solteras consideran que el sexo prematrimonial es de mayor riesgo. Mientras que los varones solteros consideran que son las caricias íntimas las que encierran un mayor riesgo. Por otro lado, aunque son pocos en número, los internautas casados, de ambos géneros, expresaron en su mayoría que son las relaciones pre matrimoniales las de más riesgo.

Vemos cómo las solteras consideran como mayor pérdida la virginidad vaginal que los varones solteros, quienes consideran como mayor riesgo el preámbulo mismo del coito, es decir, las caricias íntimas.

Esto nos permite inferir la dirección en que deben ir los programas de promoción de la sexualidad saludable, con los énfasis respectivos para solteros masculinos y femeninos.

La Tabla 4 muestra que el $30.6 \%$ de los solteros que vive con sus padres considera que el mayor riesgo son las relaciones sexuales prematrimoniales, mientras que un $30.8 \%$ de los solteros que no vive con sus padres considera que son las caricias íntimas el mayor riesgo. Esto puede ser debido a que los solteros que viven fuera del hogar paterno tienen un mayor sentido de prevención, quizá por causa de las historias o experiencias propias o vicarias que hayan tenido.

La Tabla 5 nos permiten inferir que los varones que iniciaron más tardíamente una relación afectiva consideran como mayor 


\section{Tabla 3:}

Intervalo entre sexo, estado civil y riesgos en el noviazgo

\begin{tabular}{|c|c|c|c|c|c|c|c|c|c|c|c|c|}
\hline \multirow{2}{*}{ E } & & & \multicolumn{9}{|c|}{ Riesgo en el noviazgo } & \multirow[b]{2}{*}{ Total } \\
\hline & \multicolumn{2}{|c|}{ Estado Civil } & 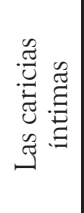 & 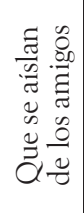 & 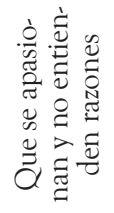 & 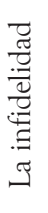 & 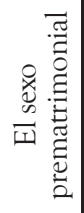 & 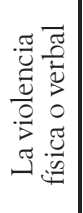 & 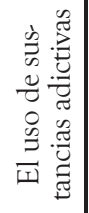 & 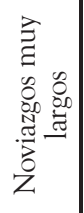 & $\stackrel{\Xi}{0}$ & \\
\hline O & & Masculino & 11 & 1 & 5 & 4 & 6 & 2 & 0 & 1 & 1 & 31 \\
\hline ڤ & $\vec{\nabla}$ & Femenino & 6 & 5 & 6 & 6 & 14 & 1 & 1 & 4 & 2 & 45 \\
\hline Total & & 17 & 6 & 11 & 10 & 20 & 3 & 1 & 5 & 3 & 76 & \\
\hline 움 & 임 & Masculino & 2 & 1 & 1 & & 5 & 1 & & 3 & & 13 \\
\hline$\tilde{\Xi}^{\mathscr{\Xi}}$ & $\stackrel{\widetilde{D}}{0}$ & Femenino & 1 & 1 & 1 & & 3 & 0 & & 2 & & 8 \\
\hline Total & 1 & 3 & 2 & 2 & & 8 & 1 & & 5 & & 21 & \\
\hline$\stackrel{\circ}{\stackrel{0}{g}}$ & : & Masculino & 1 & & & & & & & & & 1 \\
\hline Total & & 1 & & & & & & & & & 1 & \\
\hline $\begin{array}{l}\frac{0}{0} \\
. \frac{\pi}{0} \\
0 \\
.0 \\
0\end{array}$ & 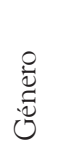 & Masculino & & & 1 & & & & & & & 1 \\
\hline Total & & & & 1 & & & & & & & 1 & \\
\hline & 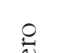 & Masculino & 14 & 2 & 7 & 4 & 11 & 3 & 0 & 4 & 1 & 46 \\
\hline 苞 & : & Femenino & 7 & 6 & 7 & 6 & 17 & 1 & 1 & 6 & 2 & 53 \\
\hline Total & & 21 & 8 & 14 & 10 & 28 & 4 & 1 & 10 & 3 & 99 & \\
\hline
\end{tabular}

riesgo las caricias íntimas, mientras que las mujeres que iniciaron entre los 15 y 18 años una relación sentimental consideran que es un mayor riesgo las relaciones sexuales prematrimoniales $(52.9 \%)$. 
Tabla 4:

Intervalo entre vive con sus padres, estado civil y riesgos en el noviazgo.

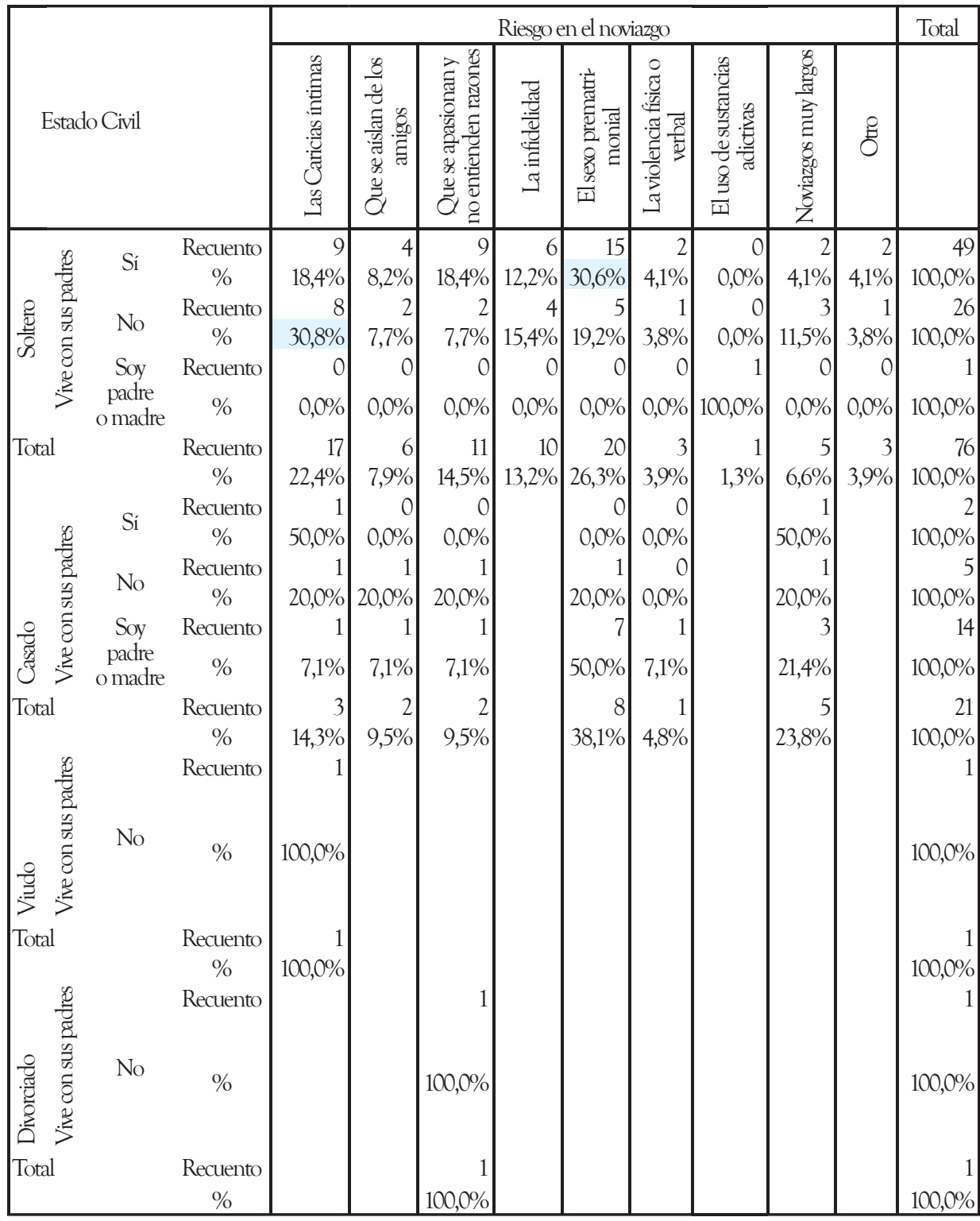




\section{Tabla 5:}

\section{Intervalo entre edad de su primera relación sentimental, riesgo en el noviazgo y sexo.}

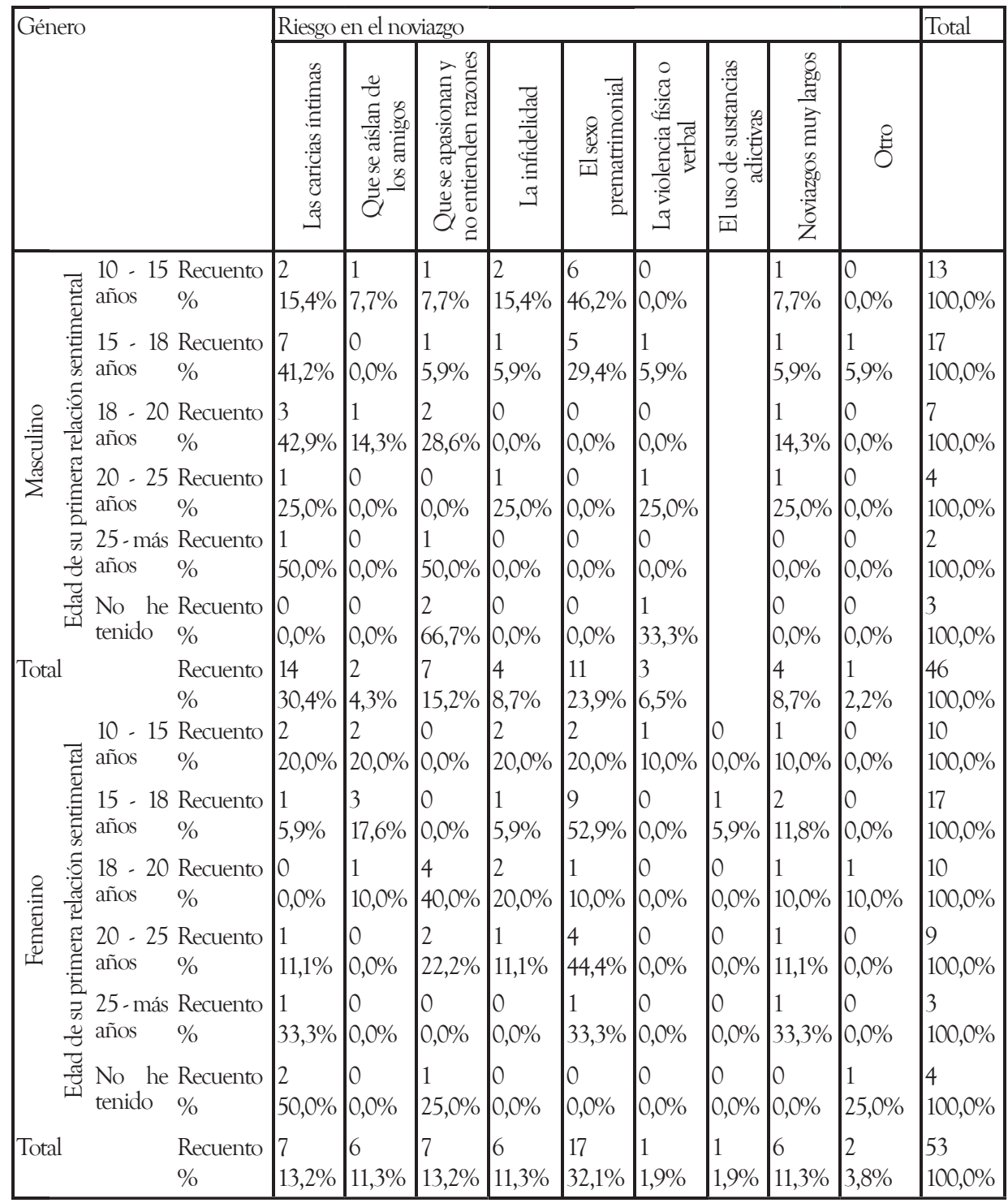




\section{Discusión}

Los resultados permiten observar que los jóvenes participantes tienen conocimiento de los riesgos del noviazgo, dando un mayor énfasis, las damas, a las relaciones sexuales coitales, y los varones a las caricias íntimas. Además, los encuestados menores de 18 consideran de más riesgo el sexo coital mientras que los mayores de 18 consideraron en su mayoría las caricias íntimas.

Fuentes, Martín \& Pérez, consideraron que las relaciones sexuales son el más alto riesgo debido a que hay intercambio de fluidos orgánicos (semen, sangre y líquidos lubricantes sexuales). Este conocimiento es más claro en las mujeres encuestadas aparentemente.

Por otro lado, están las caricias íntimas que son consideradas como el primer contacto entre dos personas que se gustan, para luego recién conocerse, siendo esta práctica ajena a la cosmovisión bíblica. Los jóvenes varones encuestados consideran que son las caricias íntimas de más riesgos, y ciertamente, éstas son previas al sexo coital.

\section{Conclusión}

Es necesario utilizar las redes sociales y Tecnologías de la Información y de las Comunicaciones (TIC's) en los esfuerzos por difundir programas y conferencias de capacitación para jóvenes en el área de sexualidad saludable y sociabilización ética y de valores.

Los programas para jóvenes y adolescentes de difusión de conductas de riesgo sexual deben tener énfasis en la orientación sexual saludable, así también en competencias sociales tales como autoestima, resiliencia y autonomía en sus decisiones saludables.

Cabe señalar que es mejor dar programas diferenciados por género, brindando un énfasis mayor en aletargar el coito en las mujeres, siento este punto aparentemente más significativo; y en varones, trabajando actitudes de autonomía en cuanto a la presión de grupo ante las caricias íntimas. 


\section{Bibliografía.}

Alonso, Macarena Blázquez, Juan Manuel Moreno Manso, y María Elena GarcíaBaamonde Sánchez. "Estudio del maltrato psicológico, en las relaciones de pareja, en jóvenes universitarios. (Spanish)". Electronic Journal Of Research In Educational Psychology 7, no. 2 (Setiembre 2009): 691-714.

Bringas Molleda, Carolina et al. "Tolerance towards dating violence in Spanish adolescents". Psicothema 24, no. 2 (Mayo 2012): 236-242.

Cerdà, Francesc Llorens, y Neus Capdeferro Planas. "Facebook's Potential for Collaborative e-Learning". RUSC: Revista De Universidad Y Sociedad Del Conocimiento 8, no. 2 (Julio 2011): 197-210.

80 Fuentes, Luzmila, Emma Martin, y Amílcar Pérez. "Prácticas sexuales de riesgo en adolescentes. (Spanish)". Informe Medico 10, no. 2 (Febrero 2008): 95-101.

González, M. Pilar et al. "Prevalence and Predictors of Sexual Aggression in Dating Relationships of Adolescents and Young Adults". Psicothema 21, no. 2 (Mayo 2009): 234-240.

Halpern-Felsher, Bonnie L., y Yana Reznik. "Adolescent Sexual Attitudes and Beha- viors: A Developmental Perspective". Prevention Researcher 16, no. 4 (Noviembre 2009): 3-6.

INEI, Encuesta Nacional de Hogares - 98, II Trimestre. INEI, http://wwwinei. gob.pe/biblioineipub/bancopub/Est/ Lib0078/S03-1.htm (consultado: 21 de julio, 2012).

Mallet, Pascal, y Dominique Herbe. "Does Knowledge about Sexuality Prevent Adolescents from Developing Rape-Supportive Beliefs?". Journal Of Sex Research 48, no. 4 (Julio 2011): 372-380.

Peltzer, Karl. "Sexuality among adolescents in rural and urban South Africa". South African Review Of Sociology 37, no. 2 (Junio 2006): 189-199.

Rojas-Solis, José Luis, y Eugenio Carpintero Raimúndez. "Sexismo y agresiones físicas, sexuales y verbales-emocionales, en relaciones de noviazgo de estudiantes universitarios" Electronic Journal Of Research In Educational Psychology 9, no. 2 (Setiembre 2011): 541-564.

Viñar, Marcelo. Mundos adolescentes y vértigo civilizatorio. Montevideo: Ediciones Trilce, 2009.

Wechsler, Howell et al. "Youth risk behavior surveillance - United States, 2011". Morbidity And Mortality Weekly Report. Surveillance Summaries (Washington, D.C.: 2002) 61, no. 4 (Junio 8, 2012): 1-162. 